\title{
An Equivalence for Both Coulombian and Gravitational Interactions
}

\author{
Carmine D’Errico \\ Technical Services Department, Belden Electronics GmbH, Neckartenzlingen, Germany \\ Email: carmine.derrico@belden.com
}

How to cite this paper: D'Errico, C. (2019) An Equivalence for Both Coulombian and Gravitational Interactions. Journal of High Energy Physics, Gravitation and Cosmology, 5, 1068-1089.

https://doi.org/10.4236/jhepgc.2019.54060

Received: March 26, 2019

Accepted: September 15, 2019

Published: September 18, 2019

Copyright (c) 2019 by author(s) and Scientific Research Publishing Inc. This work is licensed under the Creative Commons Attribution International License (CC BY 4.0).

http://creativecommons.org/licenses/by/4.0/ (c) (i) Open Access

\begin{abstract}
The main purpose of this article is to demonstrate that electromagnetic and gravitational phenomenology are two different expressions of the same interaction that we can call "universal interaction". In order to reach the aim, it is therefore necessary to imagine the matter in a slightly different way, as well as equivalent, to what has been done until today by the literature everywhere accepted. Even if differently imagined, this proposed structure of matter cannot and must not escape from reflecting the measurements and phenomenologies widely experimented in laboratories all over the world. In the proposed model the proton radius; the electron mass; the Avogadro constant; the existence and the mass of neutron and the existence of neutrino are theoretically derived. The main consequence is therefore a more general rewriting of Newton's law of universal gravitation. A definitive value for the universal gravitational constant is proposed.
\end{abstract}

\section{Keywords}

Gravitational Field, Electric Field, GUT, Classic Theory of Gravitation, Proton Radius, Neutron Radius, Avogadro Constant, Electron Mass, Neutrino Mass, Newtonian Constant of Gravitation, Language Constant

\section{Introduction}

The present paper, which is based on an application of the philosophical concept of Occam's razor to the modern questions of physics, aims to investigate and determine a logical truth in the field of modern physics. The determination of one of the possible logical truths to be studied imposes as an immediate consequence of its scientific verifiability in the sense of physical-mathematical truths. More generally, we will build an algebraic logical model through which we will be able to express simultaneously two physical truths that are still far from being 
unified: gravitational phenomenology and electromagnetic phenomenology. In order to proceed with the dissertation it is necessary to define the physical aspect of three logical-anthropological concepts: time, interaction and the speed limit.

- Time is then defined by a mechanical structure (therefore, for reasons related to our human history, a mass) incorporated into a mathematical surface that expresses the geometric concept of repetition. Technically, we will give time the philosophical structure of a recursive definition: the reoccurrence in the visual memory of a trivial geometric arrangement composed of two elementary objects.

- In defining the interaction, let's shift the analysis of the problem of unifying electromagnetism and gravity to the 17th century (let's pass the pun) in time: that is to say, we will play the role of an observer who, for the first time, measures Coulomb's experiments and Newton's experiments and wants to give to two aspects, which present themselves in history as different, the same name. The conciliatory observer decides then, being in this hypothetical XIX century, to give to the manifestation of the forces a single primordial explanation: the exchange of a momentum by the means of unknown entities. The identity of the interaction and of the entities will be illustrated in the following.

- Speed is considered as a primordial concept: once the standard time is fixed (a cyclical change of position), the space-time function of a moving body occupying different positions can be expressed in function of the standard time. When two different positions are measured, the clock strokes (amount of position changement) are counted and the speed is determined. By limit velocity we mean, because of the recursive structure with which we have defined time, that nothing can move faster than the change of position in our reference clock. As imaginable the speed limit is $c$.

In an original article by D.M. Snyder [1], the author succeeds in merging in a more general unifying consideration the curved space-time of general relativity to Lorentz's transformations of special relativity. One of the key points of the proposed observations lies in the intuition that the "[...] spacetime curvature may be developed by considering an accelerating reference frame or pattern of such reference frames as a pattern of local Lorentz frames moving at slightly different uniform, translational velocities relative to one another [...]". Quoting the main observations of the author further "[...] As noted, the derivation of the spacetime curvature associated with an accelerating reference frame may be based on the notion that an accelerating reference frame is composed of a pattern of local Lorentz frames (essentially, local, inertial reference frames) [...]". "[...] If the spacetime continuum is locally Lorentzian, and the velocity of light is therefore locally invariant and finite, then the locally invariant and finite velocity of light provides a connection between the fundamental forces of gravitation and electromagnetism [...]". Starting from these considerations, the proposed work focuses on defining as the founding stone of the equivalence between gravitational and electromagnetic forces the existence of an elementary mechanism that 
acts simultaneously as a source of field, clock and controlled microsystem that defines within itself the invariance of the speed of light.

The effective existence of an equivalence for both gravitational and coulombian interactions seems also consistent with the well known phenomenon of the gravito-magnetism [2].

\section{The Elementary “On Board" Clock}

- Let's define "primary matter" an agglomeration of any size constituted by at least one entity (a brick) primitive and indivisible (quantized) that we will call $P A N$.

- We postulate that each agglomerate of primary matter can be associated to a spherical surface $\partial m$ that circumscribes it and such that each point $a_{i}$ of the primary matter is internal to the geometric whole closed and limited by $\partial m$ :

$$
a_{i} \subset \partial m \backslash a_{i} \cap \partial m=\varnothing
$$

- Let's suppose we have an agglomerate of primary matter of any density and distribution but of determined rest mass. In its rest conditions we consider it like bounded by an ideal spherical surface of radius $2 \lambda_{0}$. We give this special agglomeration of primary matter the name of PANONE.

- The rest mass of a PANONE can have an arbitrary sign conferring to the primary matter two main characteristics:

- The amount of matter, that is the number of PANONE of which the primary matter is constituted, we can measure it in $[\mathrm{kg}]$ and its module is non-negative.

- The mass that can be also null for a not null amount of matter.

- Let's create an object made of 2 tangent PANONE that rotate with respect to a vertical axis passing through the tangential point with angular velocity $\bar{\omega}_{p}=2 \pi v_{p} \hat{\omega}_{p}$. The module of the speed of the point which is diametrically opposed to the point of tangency and lying on the ideal surface that circumscribes each of the PANONE is $v=4 \pi \lambda_{0} v_{p}$. Let's refer to this as an "elementary clock' (see Figure 1).

- In order for this clock to remain in rotation we need to hypothesize the existence of a momentum exchange between the two PANONE that we will call generically $p_{e x}$ and about which we will say nothing in this paper.

The speed of the ideal point belonging to the surface circumscribing the PANONE is $c$, verifying in this case the condition:

$$
c=4 \pi \lambda_{0} v_{p}
$$

If stimulated to move with speed any $\bar{v}$ parallel to $\bar{\omega}_{p}$ this object must not violate the fundamental principles namely:

1) The speed of the extreme point of the surface surrounding the PANONE must remain $c$.

2) The angular momentum must be preserved. 


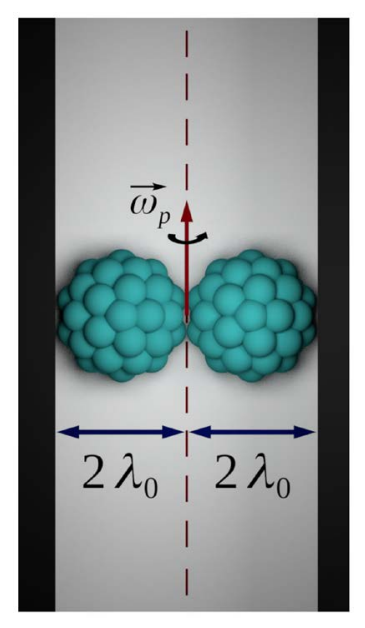

Figure 1. An artistic view of the elementary complementary clock.

The first of the two conditions imposes as a direct consequence that:

$$
v_{t}=v_{P} \sqrt{1-\frac{v_{t}^{2}}{c^{2}}}=v_{P} \gamma_{p}^{-1}
$$

This condition is the equivalent of the time dilation of Lorentz. Calculating the angular momentum $\bar{L}_{c k}$ of the elementary clock we obtain:

$$
\bar{L}_{c k}(v)=I_{c k}\left(m_{c k}\right) \bar{\omega}_{p} \gamma_{p}^{-1}
$$

where $I_{c k}\left(m_{c k}\right)$ is the inertial momentum of the elementary clock as a function of its rest mass. The relation 4 shows how, in order to preserve the angular momentum, it is necessary that the moment of inertia, i.e. that the rest mass of each of the PANONE of the elementary clock, has to be directly proportional to $\gamma$, $I_{\text {PANONE }} \propto\left(m_{\text {PANONE }} ; \gamma\right)$. Therefore, we can consider the relativistic generic mass, consistently with the special relativity [3], as:

$$
m(v)=m_{0} \gamma
$$

It might be argued that the created elementary clock needs infinite energy to be rotated around the central axis. But this is not the case because of the definitions. We can therefore generalize by saying that all the implications of special relativity are valid [4].

The surface underlying the generic PANONE due to the length contraction could be seen in a freeze image of the clock in Figure 2.

The transversal remains a circle, the longitudinal section (see Figure 2) is following:

$$
l_{t}(r)=l_{0} \gamma^{-1}= \pm \sqrt{r\left(2 \lambda_{0}-r\right)\left(1-\frac{r^{2}}{4 \lambda_{0}^{2}}\right)}
$$

\section{Presumption of Behaviour at the Boundary $\partial m$}

Let we define the surface $\partial m \in R^{3}$ like a bijective-functional-surface which works as a geometric bijective function such that 


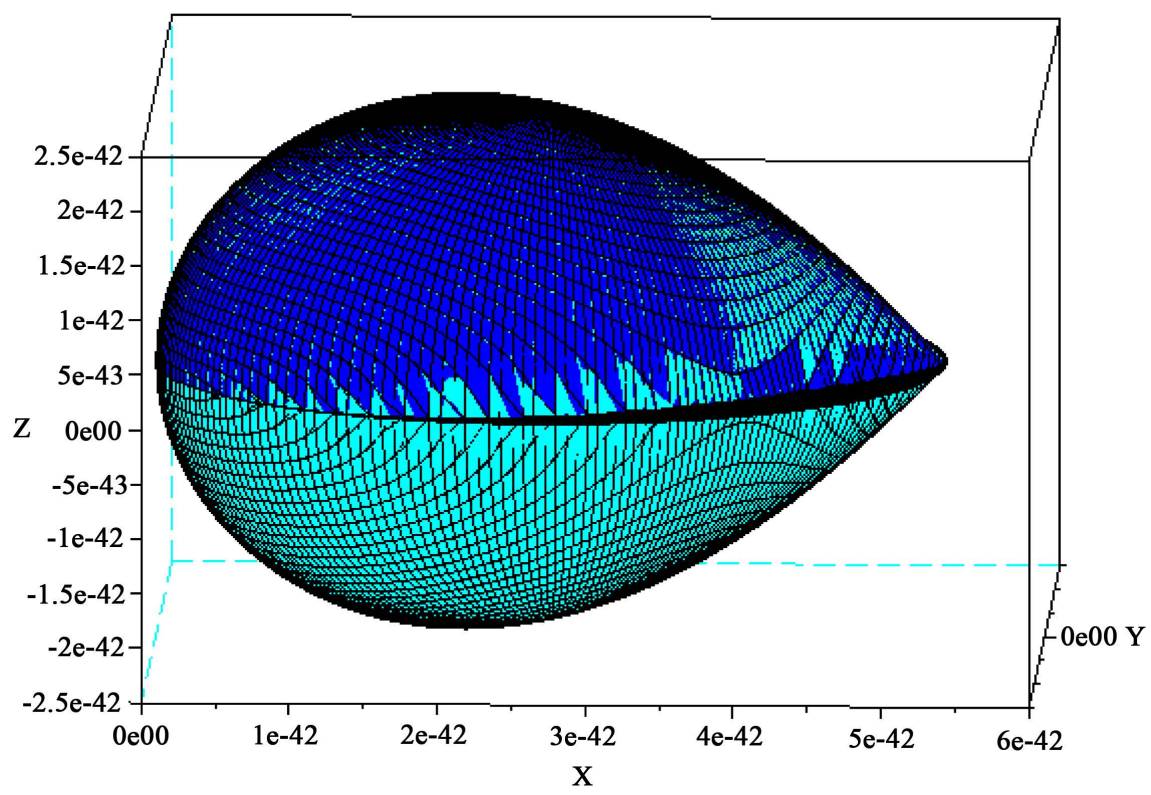

Figure 2. Mathematical 3d reconstruction of the ideal surface underlying the PANONE in an elementary clock. $\mathrm{Z}$ is the longitudinal axis in a generic freeze image. $\mathrm{XY}$ is the transversal plane.

$$
\partial m: \forall a_{i} \in m_{i} \in\left\{R^{3} \times t\right\} \leftrightarrow e_{i} \in\left\{R^{2} \times c t\right\}
$$

The frontier surface $\partial m$ transforms (see Figure 3 ) each element of the primary matter existing in space-time into a luminal entity $e_{i}$ of a special space $\left\{R^{2} \times c t\right\}$ and vice versa. For the entity that moves at luminal speed, the temporal coordinate becomes infinite and the longitudinal spatial coordinate becomes immeasurable. An observer placed in an inertial frame of reference external to the entity sees the spatial longitudinal dimension and the temporal dimension of the entity merge into a single spatial coordinate of the type $c t$. We can therefore assert that the frontier of primary matter behaves as a geometric function that applies a transformation of the space-time.

\section{The Elementary Far-Field Interactions}

Let us make the following considerations:

- We define "elementary interaction" as the interaction between two agglomerates of primary matter with dimensions considerably larger than a PANONE. In this case, two protons respectively with mass $m_{p}$ each is inscribed in a surface $\partial m$ of radius $R_{p}$ placed at a distance $r \gg 2 R_{p}$ (far field) interacting in a philosophically empty space.

- The internal mechanics of the clocks of the primary matter determines a periodic and isotropic expulsion of entities (see Figure 4) moving with speed $c$ with a frequency of $v_{p}=2 \pi \omega_{p}$;

- Each entity has a non-zero momentum equal to $\tilde{p}_{0}$;

- The mass $m_{p 1}$ emits $\aleph_{p}$ entities, a subset of which are intercepted by the boundary surface around the mass $m_{p 2}$ and vice versa. 


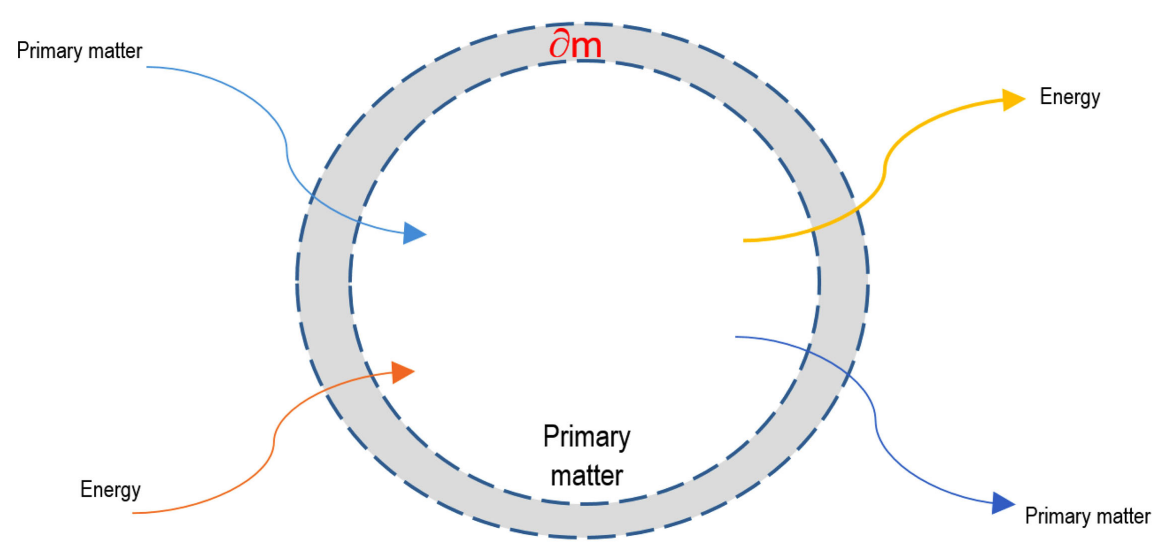

Figure 3. A representation of the "boundary function".

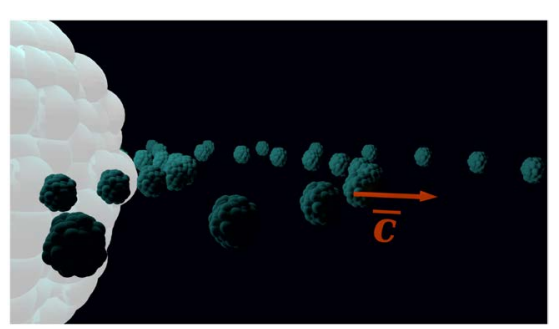

Figure 4. An artistic vision of panones emission. The figure refers only to the central planar section.

For the conservation of the momentum we can assert that the two bodies moves away from each other along the line joining the two centers of mass. The momentum $\bar{p}_{p}$ per unit of surface at a generic distance $r$ emitted by the generic conglomerate of primary matter can be expressed as follows:

$$
\bar{p}_{p}=\frac{\aleph_{p}}{4 \pi r^{2}} \tilde{p}_{0} \hat{r}
$$

where $\hat{r}$ is the radial versor outgoing from the emitting primary matter body. The variation in the momentum due to collisions intercepted by the body $m_{p 2}$ with circular section of impact $\Sigma_{p}=\pi R_{p}^{2}$ is:

$$
\Delta \bar{p}_{p}=\frac{\aleph_{p}}{4 \pi r^{2}} \tilde{p}_{0} \pi R_{p}^{2} \bullet \hat{r}
$$

By multiplying both amounts by the emission frequency $v_{p}$ end taking into account Equation (3) and the mutual effect we obtain that the total amount of momentum variation is as follows:

$$
\Delta \bar{p}_{p} \nu_{p}=\frac{\aleph_{p}}{4 \pi r^{2}} \tilde{p}_{0} \Sigma_{p} \nu_{p}\left(\gamma_{p 1}^{-1}+\gamma_{p 2}^{-1}\right) \hat{r}
$$

where $\gamma_{p 1}$ and $\gamma_{p 1}$ are the Lorentz factors for each of the emitting bodies. Equation (9) has the size of a force.

\section{The Number $\aleph_{p}$ of Emitted Entities}

Because of the invariance of charge [4] [5] and being present in the last relation 
9 exclusively constants, we are obliged to deduce that the energy of entities is inextricably linked to the energy state of the PANONEs of primary matter, only in this case it's is possible to correlate the energy of each emitted psudo-entity to the Lorentz factor of the emitting body, then the entity energy has to be wrote as $p_{0} \gamma_{p}$.

It is therefore of fundamental importance to determine the number of $\mathrm{Pa}$ nones $\aleph_{p}$ constituting the peel of the primary matter and which are affected by the geometric transformation of the boundary $\partial m$. We will hypothetize (until proven otherwise) the primary matter to be spherical with radius $R_{p}=2 n_{p} \lambda_{0}$ with $n_{p} \gg 1$. In the simplest image of an isotropic emission with spherical symmetry we consider to be exactly $2 \lambda_{0}$ the thickness of the "zest" more external than the sphere of primary matter (Panones diameter). $\frac{4 \pi}{3} \lambda_{0}^{3}$ is the volume of a single Panone. The volume of primary matter decreases by a quantity $V_{d}$, due to the peel-off of the issued $\aleph_{p}$ entities, for an overall amount equal to:

$$
V_{p}^{\text {emitted }}=\frac{4}{3} \pi\left[R_{p}^{3}-\left(R_{p}-2 \lambda_{0}\right)^{3}\right]=\frac{4}{3} \pi\left(6 R_{p}^{2} \lambda_{0}-12 R_{p} \lambda_{0}^{2}+8 \lambda_{0}^{3}\right)
$$

Since $R \gg 2 \lambda_{0}, V_{p}$ can be roughly considered as:

$$
V_{p}^{\text {emitted }} \cong 8 \pi \lambda_{0} R_{p}^{2}
$$

The $\aleph_{p}$ number can simply be calculated using the $V_{d}$ definition of the volume occupied by only one Panone:

$$
\aleph_{p}=\frac{V_{p}^{\text {emitted }}}{V_{\text {PANONE }}}=\frac{8 \pi \lambda_{0} R_{p}^{2}}{\frac{4}{3} \pi \lambda_{0}^{3}}=24 n_{p}^{2}
$$

The number of issued entities is an even number.

\section{An Interpretation of the Fine Structure Constant and Planck's Constant}

For each of the agglomerations of primary matter from which the luminal entities protrude it is possible to define a surface $S=\partial m$ that encompasses its material extension and calculate the flow of this volumic energy through it as follows:

$$
\oint_{S} \frac{\aleph_{p}}{4 \pi r^{2}} p_{0} v_{p} \hat{r} \cdot \hat{n} \mathrm{~d} S=\aleph_{p} p_{0} v_{p}
$$

We observe that the flow of a volumic radiating energy through a surface external to its material distribution is the origin of a force field that we will assume, in the following, to be exactly the coulombian force field. We will not express this relation in local form as it is not possible to consider the source as punctiform.

From this observation it transpires that the sources of the field are describable as a volumic radiating energy, i.e. there is a substantial equality between mass 
and energy, which cannot be separated from the propagation of energy waves. In the following part of the paper we will deal exactly with these topics.

We can then impose the 9 as following:

$$
\Delta \bar{p}_{p} v_{p}=\frac{\aleph_{p}}{4 \pi r^{2}} \tilde{p}_{0} \Sigma_{p} v_{p}\left(\gamma_{p 1}^{-1}+\gamma_{p 2}^{-1}\right) \hat{r} \triangleq \frac{e^{2}}{4 \pi \varepsilon_{0}} \frac{\hat{r}}{r^{2}}=\bar{F}_{C}
$$

From this last relation it emerges that the charge would not seem to be an invariant quantity but this is not possible [4] [6]. We can therefore imagine that each emitted entity corresponds to a material PANONE inside to the surface $\partial m$ (therefore subject to the laws of the SR).

It therefore makes sense to believe that this phenomenon, which combines electromagnetism and electroweak force, originates from an unfathomable phenomenon of dispersion of the outer peel of the material from which it originates. Using the Sommerfeld notation for the coulombian interaction [7] and because of Equation (14) we derive a new expression of Planck's constant:

$$
h \triangleq \frac{\aleph_{p}}{2 \pi} \frac{p_{0}}{\alpha c} \Sigma_{p} v_{p}
$$

This last equation shows that the relativistic mass of the emitting body and the momentum $p_{0}$ of the emitted entities linked each other and proportional to $\gamma_{p}$. In the above relation we just used the symbol $\triangleq$ in order to remark that the hypothesis is the boundary behavior. Then we can now write:

$$
h \triangleq \frac{\aleph_{p}}{2 \pi} \frac{m_{0}}{\alpha} \Sigma_{p} v_{p}
$$

where we considered $\frac{p_{0}}{c} \triangleq m_{0}$. In this way we defined the Planck's constant as function of other natural constants. This last equation is valid also for the relativistic expression of the couple $\left\{\frac{m_{0}}{\alpha} ; v_{p}\right\}$ but you must consider $\Sigma_{p}$ as the transverse surface of the moving body. The $\frac{m_{0}}{\alpha}$ ratio indicates that a $\mathrm{Pa}$ none is a set of $\alpha^{-1}$ most elementary particles. To each of these particles we give the name of PAN.

We can therefore give a more precise microscopic interpretation of the Sommerfeld constant and Planck constant:

- $\aleph_{p}$ is the number of emitted entities for each expulsion or the number of a Panones on the peel of a proton.

- $\alpha^{-1}$ is the average number of PAN of which a PANONE is composed;

- so $\frac{m_{0}}{\alpha}$ is the mass of the Panone and $m_{0}$ is the mass of the PAN (the subpart of Panone);

- $\lambda_{0}$ is the radius of the Panone;

- $v_{p}$ is the characteristic emission frequency for a proton and the rotation frequency of the elementary clock.

- $c$ is the maximum speed of a point on the sphere of the Panone and the ve- 
locity at which an entity propagates;

- $\Sigma_{p}$ is the cross section of a proton to the rest.

The Planck constant as well as in Equation (16) alone is sufficient to express the entire behaviour of the classic fundamental interactions interpreted by this model. Equation (16) report alone could be defined as the universal relation of electrostatic interactions in far field. In the near field, careful calculations must be made to evaluate the non-linearities.

\section{Verification of the Model through Determination of Proton Charge Radius}

The determination of the proton charge radius is one of the open questions of modern physics [8]. The literature, which documents several laboratory experiments, contains tests on alternative theoretical approaches to the problem. The experiments of through the muon-hydrogen spectroscopy of Antognini have detected a value equal to 0.84184 [fm] [9] and 0.84087 [fm] [10], on which the results of Beyer [11] are very close with a radius equal to 0.8335 [fm]. The framework of the experimental data is polarized instead around an average value of about 0.88 [fm] right through many experiments performed over the past 50 years with Electron scattering measurements [12] [13] [14] and Laser spectroscopy of atomichydrogen measurements using QED theory.

In a very recent as well as beautiful article by Miller, the author discusses how it is not possible to define a spherical shape for the charge radius of the proton even if one can identify its precise spatial density [15].

The here proposed work of investigation, through different tools and breaking with traditional and modern frameworks, starting from definitions and premises completely different from the theoretical ones used by Miller, aims to trace a new path of research which tries to answer to a subset of opened questions that have not yet been answered. The following theoretical prediction is reflected in the current experimental results. We will therefore allow ourselves to continue to use the concept of "frontier of matter" as defined above, reserving for ourselves, in the following, the right to define it further.

Directly from Equation (15), by writing $R_{p}=2 n_{p} \lambda_{0}$ and considering a natural mass density $\rho_{0}=\frac{m_{0}}{\alpha V_{P A N O N E}}$ we can write:

$$
h=\left(\aleph_{p} \frac{m_{0}}{\alpha}\right) \Sigma_{p} v_{p}=\left(8 \pi R_{p}^{2} \lambda_{0} \rho_{0}\right)\left(\pi R_{p}^{2}\right) v_{p}=\frac{3}{2}\left(\frac{4}{3} \pi R_{p}^{3} \rho_{0}\right) R_{p}\left(4 \pi \lambda_{0} v_{p}\right)
$$

By multiplying and dividing by $\frac{2}{3}$ and because of the definition Equation (2) we can write:

$$
h=\frac{3}{2} m_{p} R_{p} c
$$

where $m_{p}$ is the mass of primary matter and $R_{p}$ is its radius. We can therefore make explicit the radius of primary matter taken under examination as follows: 


$$
R_{p}=\frac{2}{3} \frac{h}{c \cdot m_{p}}=0.88093990250 \times 10^{-15} \mathrm{~m}
$$

The radius expression thus calculated is fully consistent also with the CODATA [16] values for the proton radius with a standard relative uncertainity of $6 \times 10^{-3}$. It has to be observed that the calculated value coincides exactly with the values of the proton charge radius calculated with ep-scattering and spectroscopy.

From Equation (18) it emerges that Planck's constant contains within itself, in addition to the propagative model, the physical structure of matter itself.

Due to uncertainties linked to the mass of the proton still under investigation, we could fix the uncertainties on Planck's constant, on the mass and radius of the Proton in this way:

$$
\frac{h}{m_{p} R_{p}}=\frac{3}{2} c
$$

Since Planck's constant is invariant and must be the same for each charge iteration, we can estimate that the radius $R_{p}$ is the charge radius not only for the proton but of each electric charge. Consequently also the frequency $v_{p}$ and the number of PANONEs released in each emission $\aleph_{p}$ remain invariant. This type of relations adopted does not clarify the internal structure of the surface $\partial m$. These considerations about the physical nature of the proton certainly fit into the still completely open debate in the physics world about the proton radius puzzle [6] and the proton spin crisis [17].

\section{The Gravitational Equivalence: From Relativistic Mass-Energy Relation to Avogadro's Constant and Electron Mass}

In this section of the proposed research the principal hypothesis is made. The history of humanity is evolved by successive advances and new concepts are constructed on the foundations of the old ones. In the following, we make a very strong historical-anthropological assumption: humans refer to all that appears different by different names. And for each of the different things that he sees and experiences he creates models. Humans write their models in the logical-mathematical language: the only one they know, so that they can understand and represent them in the subspace of their perceptions. The external reality is therefore not knowable in its entirety, but exclusively observable within the subspace of human perceptions in which it is projected.

In this specific case we will consider that gravitational and electromagnetic effects are two different manifestations of the same universal interaction evolved historically both independently. If the two models are equivalent then there must exist a constant that correlates them, which we will call language constant. The correlation constant between two equivalent models of which the observables are completely different (masses and charges) by the will of the ancient observers cannot and must not be any number. The language constant needs to 
be a special number, that is unitary, and its units of measurement must reflect in themselves the characteristics of logic and language.

By using the proton radius relation 18 we can obtain the following mass-energy relation:

$$
E_{P}^{0}=h \frac{4}{3} \pi \frac{v_{p}}{n_{P}}=m_{p} c^{2}
$$

where $E_{p}^{0}$ is the rest energy of a proton with rest mass $m_{p}$. Directly from 21

$$
\hbar \alpha c=\left(\frac{3 \alpha \lambda_{0}}{2 \pi m_{p}} c^{2}\right) n_{p} m_{p}^{2}
$$

The member to the left of the last equation (less than $R^{2}$ ) represents the expression of the coulombian force using the Sommerfeld relations. The one to the right is certainly the Newtonian interaction. We can write both equations then in vector form as follows:

$$
\hbar \alpha c \frac{\hat{r}}{r^{2}}=\left(\frac{3 \alpha \lambda_{0}}{2 \pi m_{p}} c^{2}\right) n_{N} m_{p}^{2} \frac{\hat{r}}{r^{2}}
$$

where we use $n_{N}$ to discriminate it from $n_{P}$ and separate the respectively gravitational and electromagnetic cases. We can now define

$$
G \triangleq\left(\frac{3 \alpha \lambda_{0}}{2 \pi m_{p}} c\right) \frac{\xi}{25} \cdot 1[\mathrm{~mol}]
$$

where $G$ is the Newtonian constant of gravitation and $\xi$ is an appropriate constant measured in [m/s.mol]. Using the definition 24 in Equation (22) we are able to calculate the following quantity:

$$
\hbar \alpha c=25 G c m_{p}^{2} \frac{n_{N}}{\xi} \cdot 1\left[\mathrm{~mol}^{-1}\right]
$$

It's simple to verify that:

$$
\frac{n_{N}}{\xi} \cdot 1\left[\mathrm{~mol}^{-1}\right]=\xi^{-1}\left(2 \frac{N_{A}}{\alpha}\right)=\frac{\hbar \alpha}{G\left(5 m_{p}\right)^{2}}
$$

where $N_{A}$ is the Avogadro constant. We can calculate now $\xi$ as following: $\xi=N_{A} \frac{50 G m_{p}^{2}}{\hbar \alpha^{2}}$.

The relation 24 we introduced the gravity assuming that the large masses are made up of only protons, completely neglecting other particles. We give now the language constant the symbol $\xi_{0}$ entrusting it with a value equal to $\xi_{0}=1\left[\mathrm{~m} \cdot \mathrm{s}^{-1} \cdot \mathrm{mol}^{-1}\right]$. We can calculate the relation between our purposed value and the one from relation:

$$
\frac{\xi}{\xi_{0}}=1.0011548774
$$

this ratio has to be proportional to the amount of matter that deviates from that of the proton, then we can write: 


$$
\frac{\xi}{\xi_{0}}=\left(\frac{m_{p}}{m_{p}+m_{x}}\right)^{2}
$$

where we called $m_{x}$ the mass of the unknown particle. Then the unknown is following:

$$
m_{x}=m_{p}\left(\sqrt{\frac{\xi_{0}}{\xi}}-1\right)
$$

The following Table 1 shows the values of the most important and accredited $G$ measurements.

Removing the maximum and minimum values from Table 1 the average value $G_{\text {mean }}=6.67363641 \times 10^{-11}\left[\mathrm{~N} \cdot \mathrm{m}^{2} \cdot \mathrm{kg}^{-2}\right]$. If we use the CODATA value of $\mathrm{G}$ in the relation 29 we obtain $m_{x}=-9.6500088215 \times 10^{-31} \mathrm{~kg}$. If instead of the CODATA value we use the calculated average value, we get $m_{x}=-9.0905741187 \times 10^{-31} \mathrm{~kg}$. We have to observe that:

- The searched mass is negative;

- It results $m_{X} \cong-m_{e}$, where $m_{e}$ is the CODATA mass for the electron.

We note that the mass not introduced in the a priori model is also linked to an electrical balance. An imbalance of charges in the universe would compromise the entire physical-chemical balance and, at the same time, falsify the whole of physics as it is known today. Therefore we suggest to adopt universally the following value of the universal gravitation constant:

Table 1. The most important measurements of the Newtonian constant of gravitation, G and its mean value calculated excluding the MIN and MAX values.

\begin{tabular}{cc}
\hline Reference & $G\left[\mathrm{~N} \cdot \mathrm{m}^{2} / \mathrm{kg}^{2}\right]$ \\
\hline $\operatorname{Li}[18]$ & $6.67448400 \times 10^{-11}$ \\
Li [18] & $6.67418400 \times 10^{-11}$ \\
Luther [19] & $6.67260000 \times 10^{-11}$ \\
Karagioz [20] & $6.67290000 \times 10^{-11}$ \\
Gundlach [21] & $6.67421500 \times 10^{-11}$ \\
Bagley [22] & $6.67400000 \times 10^{-11}$ \\
U. Kleinevo $\beta[23]$ & $6.67422000 \times 10^{-11}$ \\
Armstrong [24] & $6.67387000 \times 10^{-11}$ \\
Luo [25] & $6.66990000 \times 10^{-11}$ \\
Schlamminger [26] & $6.67425200 \times 10^{-11}$ \\
Luo [27] & $6.67349180 \times 10^{-11}$ \\
Parks [28] & $6.67234000 \times 10^{-11}$ \\
Quinn [29] & $6.67545180 \times 10^{-11}$ \\
Rosi [30] & $6.67191990 \times 10^{-11}$ \\
Newman [31] & $6.67435000 \times 10^{-11}$ \\
CODATA & $6.67408310 \times 10^{-11}$ \\
Mean value & $6.67363641 \times 10^{-11}$ \\
\hline
\end{tabular}




$$
G_{0}=6.6736514321 \times 10^{-11}\left[\mathrm{~N} \cdot \mathrm{m}^{2} / \mathrm{kg}^{2}\right]
$$

The proposed value with a relative standard deviation of $2.25 \times 10^{-6}$ in relation to $G_{\text {mean }}$ appears to be strongly compatible with all laboratory measurements mentioned here.

We can furthermore estimate the exact value of the Avogadro constant:

$$
N_{A}=\frac{\xi_{0} \hbar \alpha^{2}}{50 G_{0}\left(m_{p}-m_{e}\right)^{2}}
$$

and the mass of protons and electrons can be calculated as following:

$$
m_{p}-m_{e}= \pm \sqrt{\frac{\xi_{0} \hbar \alpha^{2}}{50 N_{A} G_{0}}}
$$

This equation indicates that the solutions of the model are two (see Figure 5): a universe and an anti-universe dominated by a repulsive gravitational force.

Finally, we can express the equivalence between the coulombian and gravitational models in terms of equality between charge and mass as follows:

$$
e= \pm 10\left(m_{p}-m_{e}\right) \sqrt{G_{0} N_{A} \varepsilon_{0} \frac{c}{\xi_{0}} \frac{2 \pi}{\alpha}}
$$

\section{Conclusions}

Once a language has been defined (symbols and syntax), the relation between space and time (speed) of the observed entity (Panone, $\mathrm{mol}^{-1}$ ) is an invariant. The invariance of the speed of light in all the reference frames then appears only as an exclusive consequence of the invariance of the characteristics of language.

In order to understand the world in a logical form, it must be possible to describe it in a language made up of symbols and rules. In the case of physics, the

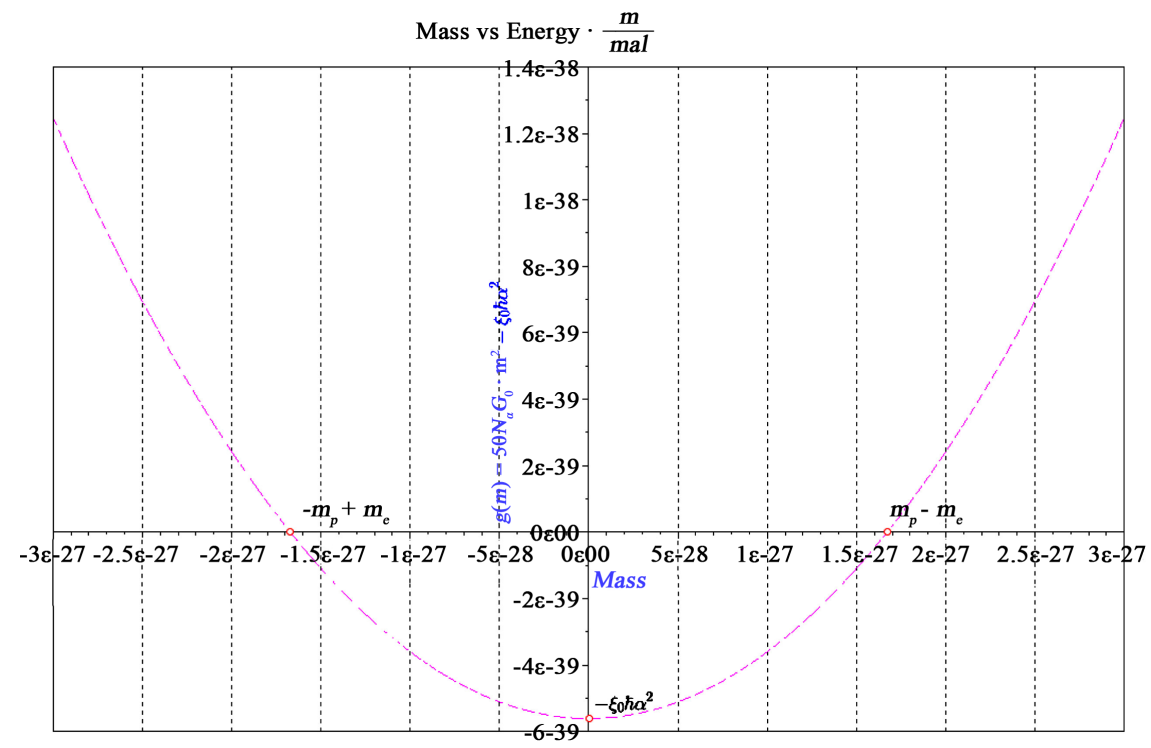

Figure 5. Matter/antimatter solutions. 
common rules are those of the fundamental algebraic system. Symbols differ according to the history of the human development, which has found different concepts for different apparent phenomena involving objects which humans gave different names and phonetics. In this paper we have shown that with a banal algebraic system it is possible to find a set of primordial symbols capable of speaking all languages when they are connected to each other by the same algebra. This not only means that all statistical, differential and integral structures are a linear combination of an elementary algebra, but it also states that the Gödel [32] principle applies to physics in its entirety. It should be noted that the entire work of Wittgenstein [33] is also fundamental to stealing the details of all the reasoning so far put forward.

Now we can derive the values of $\lambda_{0}$ and $v_{p}$. The system of unknowns describing the purposed model is now complete and by using the definition 24 we obtain:

$$
\left\{\begin{array}{l}
v_{p}=\frac{3 \alpha}{200 \pi^{2}} \frac{c^{2} \xi_{0}}{G_{0}\left(m_{p}-m_{e}\right)} \\
\lambda_{0}=\frac{50 \pi}{3 \alpha} \frac{G_{0}\left(m_{p}-m_{e}\right)}{c \xi_{0}} \\
n_{N}=2 \frac{N_{A}}{\alpha} \\
\frac{m_{0}}{\alpha}=\frac{m_{p}}{8 n_{N}^{3}}
\end{array}\right.
$$

\subsection{The Peculiarity of the Matter Structure}

We have discovered that the structure of matter (see Figure 6) is made up of elementary particles that agglomerate with each other, following a similar pattern.

Then we can solve (see Table 2) completely our system of unknown.

We found new definitions for many natural constants (see Table 3).

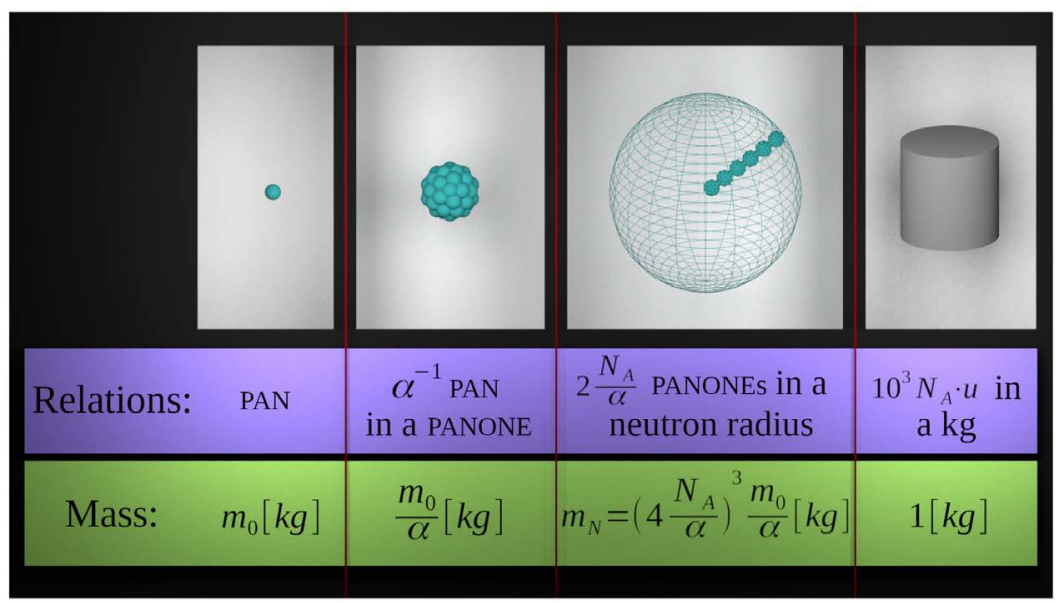

Figure 6. The nature and its natural algorithms. 
Table 2. The solutions of our unified model.

\begin{tabular}{cccc}
\hline Name & Value & Units & Description \\
\hline$v_{p}$ & $8.9345676116 \times 10^{48}$ & $\mathrm{~s}^{-1}$ & Proton Emission Frequency \\
$\lambda_{0}$ & $2.6701600831 \times 10^{-42}$ & $\mathrm{~m}$ & Panone's transversal radius \\
$n_{p}$ & $1.6505001786 \times 10^{26}$ & & Number of Panones in a proton radius \\
$\frac{m_{0}}{\alpha}$ & $4.6500868507 \times 10^{-107}$ & $\mathrm{~kg}$ & Panone's rest mass \\
\hline
\end{tabular}

Table 3. The most important results of our model.

\begin{tabular}{ccc}
\hline Name & Definition & Description \\
\hline$\xi_{0}$ & $\xi_{0}=1[\mathrm{~m} / \mathrm{s} \cdot \mathrm{mol}]$ & Language constant \\
$h$ & $\triangleq \frac{\aleph_{p}}{2 \pi} \cdot \frac{m_{0}}{\alpha} \Sigma_{p} v_{p}$ & Planck constant \\
$R_{p}$ & $R_{p}=\frac{2}{3} \frac{h}{c \cdot m_{p}}$ & Proton radius \\
$G_{0}$ & $G_{0}=6.6736514321 \times 10^{-11}\left[\mathrm{~N} \cdot \mathrm{m}^{2} / \mathrm{kg}^{2}\right]$ & Newtonian constant \\
$N_{A}$ & $N_{A}=\frac{\xi_{0} \hbar \alpha^{2}}{50 G_{0}\left(m_{p}-m_{e}\right)^{2}}$ & Avogadro constant \\
$m_{e}$ & $m_{e}=\mp m_{p} \pm \sqrt{\frac{\xi_{0} \hbar \alpha^{2}}{50 N_{A} G_{0}}}$ & Electron/Positron mass \\
$e$ & $e= \pm 10\left(m_{p}-m_{e}\right) \sqrt{G_{0} N_{A} \varepsilon_{0} \frac{c}{\xi_{0}}} \frac{2 \pi}{\alpha}$ & Elementary charge
\end{tabular}

The invariance of the language constant and the mechanism of the behaviour of the entity on the boundary can be erected to first principles that can be observed experimentally. We could define them as "fundamental behaviors" or "primordial behaviors" of entities. We have therefore demonstrated that the principle of mass conservation, the principle of energy conservation, the principle of conservation of the angular momentum, the principle of conservation of the momentum are all simultaneously verified and contained by the principle of the invariance of the constant of language in all reference systems. The direct consequence of what has been said is that there is no need for axioms. We only need the properties of the space in which we define the mechanisms of frontier that do not necessarily have to be expressed in a Euclidean geometry.

\subsection{Reshaping the Newton's Law of Universal Gravitation}

We could finally extend the Newtonian law to the microscopic world and rewrite it as following:

$$
\bar{F}_{G}=-G_{0} \frac{m_{1} m_{2}}{r^{2}} \hat{r}
$$


where the generic mass $m_{i}$ has to be considered $m_{i}= \pm n_{i} \sqrt{50 \frac{N_{A}}{\alpha} \frac{c}{\xi_{0}}}\left(m_{p}-m_{e}\right)$ with $\pm n_{i} \in \mathbb{N}$ where:

- the negative signum is to be used for antimatter;

- the positive signum is to be used for matter.

It appears that between matter and antimatter the gravitational force is repulsive. Then we can write e more general law of gravitation:

$$
\bar{F}_{G}=-n_{12} \cdot G_{0} \frac{c}{\xi_{0}}\left(2 \frac{N_{A}}{\alpha}\right)\left[5\left(m_{p}-m_{e}\right)\right]^{2} \frac{\left(\overline{r_{2}}-\overline{r_{1}}\right)}{\left|\bar{r}_{2}-\bar{r}_{1}\right|^{3}}
$$

where $\overline{r_{2}}-\bar{r}_{1}$ is the vector distance between the interacting masses.

\subsection{Progressive Entities with Negative Momentum}

When we say that "opposite charges attract each other" it is precisely because the mass of one of them is negative so the body of negative mass hit by the entity regresses in the opposite direction.

When we say "two bodies of the same charge sign reject each other" means that the entities of the electric field carry with them the information about the primary matter which generated them, so that they have a negative momentum if the mass which generated them is negative. It is also observed that the Lorentz dilatation in order to express itself requires that clocks are always oriented perpendicular to the motion of the primary matter. Then we can write the momentum of a entity or of the entity as following:

$$
\bar{p}_{0}=\left\{\hat{v}_{i} \bullet \hat{\omega}_{p}\right\} p_{0} \hat{r}
$$

where $\left\{\hat{v}_{i} \bullet \hat{\omega}_{p}\right\}$ is the scalar product between the speed versor of a general emitting body and the angolar momentum's versor of its clocks. $\hat{r}$ is the propagation speed versor of the entity. Then $\bar{p}_{0}$ is positive for matter and negative for antimatter. Therefore, the mass of a Panone is directly related to its behaviour in terms of clock. If we define in this way the elementary momentum, its determination becomes ambiguous for bodies in absolute state of stillness. Here we implicitly mean that $\hat{v}_{i}$ and $\hat{\omega}_{p}$ are only parallel or antiparallel.

\subsection{From Gravitation Law to Neutron Composition and Neutrino Mass}

Propagating the result of Equation (36) until its origin (Equation (19)) we obtain the expression of the radius of an agglomerate of primary matter globally neutral (null charge). But this is surely the neutron radius:

$$
R_{N}=\frac{2}{3} \frac{h}{c \cdot\left(m_{p}-m_{e}\right)}=8.814199387975 \times 10^{-16}[\mathrm{~m}]>R_{P}
$$

Considering the natural density like in the 17 as $\rho_{0}=\frac{3 m_{p}}{4 \pi R_{p}^{3}}$ and inserting it in the case of the neutron radius, the amount of matter difference between the 
proton and the neutron is equal to :

$$
\begin{aligned}
\Delta m_{N} & =\rho_{0} \frac{4 \pi}{3}\left(R_{N}^{3}-R_{P}^{3}\right)=m_{p} \frac{m_{p}^{3}-m_{N}^{3}}{m_{N}^{3}} \\
& =2.735794447657 \times 10^{-30}[\mathrm{~kg}] \cong 3.00 \cdot m_{e}
\end{aligned}
$$

where $m_{e}$ is the CODATA electron mass. Since we made a distinction right from the beginning between mass and primary matter agglomerates, the best result of the model emerges here overwhelmingly. In a neutron there is a proton, an electron and then again an electron and a positron. The pair $\left(e^{+}+e^{-}\right)$has zero mass but the amount of matter that constitutes them is not zero and occupies a clear and defined volume. This simple and powerful calculation expresses in an incontrovertible way and in perfect agreement with the current knowledge [34] that there is in the neutron a neutrino with an amount of matter up to 1.024 $\mathrm{GeV} / \mathrm{c}^{2}$. This further powerful result is in perfect agreement with the models currently accepted for the free neutron decay.

The description of the subatomic universe inherent in neutrinos is still in the experimental phase and the challenge for a wider knowledge of a fundamental model of interactions seems to be completely open [35]. Our model, besides being in agreement with all the experimental data currently available, seems to suggest a new approach to physics on an atomic scale.

\subsection{Simplified Solutions for the System}

Following the system of simplified solutions using the neutron mass:

$$
\left\{\begin{array}{l}
v_{p}=\frac{3}{2} \frac{N_{A}}{\pi \alpha} \frac{m_{N} c^{2}}{h} \\
\lambda_{0}=\frac{\alpha h}{6 N_{A} m_{N} c} \\
n_{N}=2 \frac{N_{A}}{\alpha} \\
\frac{m_{0}}{\alpha}=\frac{m_{N}}{8 n_{N}^{3}}
\end{array}\right.
$$

\section{Final Comments about the Proposed Framework}

The proposed theoretical framework shows the potential to be able to adopt a unified approach for the description of gravitational and electrostatic phenomena in far field until now considered irreconcilable.

All the experimental results currently achieved in the field of physics are preserved unaltered in the context of the proposed theoretical approach and no hypothesis made contradicts either classical theories or modern theories that remain alternatively valid.

The illustrated theoretical system is able to demonstrate for the first time by purely theoretical paths the existence of natural structures of primary importance given as axiomatic by the currently accepted theories (existence and mass 
of the electron, of the neutron and of the neutrino) or known by exclusively experimental means (the Avogadro constant).

Nowadays the generic concept of mass and charge are still very elusive, despite their apparent simplicity they conceal considerable pitfalls and it is extremely easy to fall into banalities or tautologies. The discussions and decisions that have been expressed, for example, in resolution 1 of the 26th GFCM (2018) [36] are "revolutionary" and attempt to fill these kinds of gaps. In this specific case, it was thought to correlate the whole SI to Planck's constant, but to do this they were forced to precipitate and clumsily and dogmatically fix Avogadro's constant (I cite: "[...] The mole, symbol mol, is the SI unit of amount of substance. One mole contains exactly $6.02214076 \times 10^{23}$ elementary entities. This number is the fixed numerical value of the Avogadro constant, $N_{A}$, when expressed in the unit $\mathrm{mol}^{-1}$ and is called the Avogadro number. The amount of substance, symbol $n$, of a system is a measure of the number of specified elementary entities. An elementary entity may be an atom, a molecule, an ion, an electron, any other particle or specified group of particles. [...]"). The theory that we propose here, instead, opens a huge and important door within the general conception of the study of physics by placing a fundamental emphasis precisely on these themes of great conceptual relevance and of general (and not special) interest.

The proposed model offers a streamlined numerical processing compared to the models currently in force, for example it is extremely simple to process problems to many bodies with a simple algebraic model of equations to differences by calculating the resultant $\mathrm{k}$-th step of the momentum. The overall model is therefore not only simplified but also less axiomatic with regard to the transformations of mass into energy and vice versa.

The further, currently tortuous, explanations about the internal structures of matter can be considered as alternatives and equivalents.

In our case, instead, by giving geometric definitions to the primary matter, it is possible to give to the charge a material consistency and a physical space that allow to find a link between the two main phenomenologies.

A further point in favour of the proposed framework is that it weaves into a single concept most of the fundamental constants of physics, providing fundamental meanings and offering inextricable bonds that correlate them all at the same time.

To a careful eye it will not have missed that the modeling of the frontier of matter in \$3. coincides with the definition of entanglement and that the elementary clock defined in Equation (2) leads directly to a form of Heisenberg's uncertainty principle. It is established that spontaneous emission, the entanglement and Heisenberg's uncertainty principle (elementary clock) are the basic principles from which the entire order of nature spreads out. In accordance with the definitions of "frontier of primary matter" phenomena such as "photonic upconversion" and phononon generation are a direct consequence. 
From the model emerge also two further characteristics of matter absolutely not negligible. The first characteristic is that the weak interaction is a consequence of the universal interaction. The second characteristic is that the strong interaction is useless because it is described by the model itself.

A last and important step taken by this framework is the relevance of the language and how once a language is chosen for the description of a problem, the solutions become a formal logical expression developed within the language itself. The critical analysis that gave birth to this paper is therefore extremely philosophical: is it possible to unify equations that describe solutions to problems formalized by different people, in different epochs with different languages and formalizations? Is it necessary to choose a new, more universal language to describe all physics in a single all-inclusive system? Is the language itself able to reveal all the truths at the same time? Are the different solutions expressed in the individual languages all equivalent and illuminate different aspects of reality? Is it necessary to have a single theory to describe the whole of physics or is it necessary to have infinite theories, all of which are irreconcilable, which together give an all-encompassing description of the truth of the facts, that is, those which Wittgenstein defined as "Tatsache"?

The limit of the physical world is nothing more than its representation in the subspace of our imagination and its acceptance is only the "how" this neural representation is reproducible through physical experiments. Choosing a traditional or an alternative path turns out to be onerous only if we analyze the dichotomy in the time domain, but if the universe is timeless we must then have confidence in the visions that leave unchanged and incorporate the physical phenomena accepted and experienced. This has been taught by Galileo, Newton, Cauchy, Maxwell, Einstein, Heisenberg, Gödel, Wittgenstein and many others.

Further developments and contributions of the scientific community in this field could lead to the development of equivalent Maxwell equations for the gravitational field capable of describing an "electrogravitational” model capable of proving equivalent to the model of general relativity.

Proceeding through the contribution of the international community in this direction could certainly be thrilling.

\section{Acknowledgements}

The original drawings and 3D graphic animations are by Davide Bazzana.

Of this writing I would like to pay homage to my inspiring Muses (solitude, music, the Enlightenment) and my wife's patience.

\section{Conflicts of Interest}

The author declares no conflicts of interest regarding the publication of this paper.

\section{References}

[1] Snyder, D.M. (2000) A Connection between Gravitation and Electromagnetism. 
[2] Corda, C. (2011) Precise Response Function for the Magnetic Component of Gravitational Waves in Scalar-Tensor Gravity. Physical Review D, 83, Article ID: 062002. https://doi.org/10.1103/PhysRevD.83.062002

[3] Einstein, A. (1905) Zur elektrodynamik bewegter koerper. Annalen der Physik, 322, 891-921. https://doi.org/10.1002/andp.19053221004

[4] Pauli, W. (2008) Teoria della relativita. Bollati Boringhieri, Torino.

[5] Ivezic, T. (1992) The Definitions of Charge and the Invariance of Charge. Physics Letters A, 162, 96-102. https://doi.org/10.1016/0375-9601(92)90983-S

[6] Carlson, C.E. (2015) The Proton Radius Puzzle. Progress in Particle and Nuclear Physics, 82, 59-77. https://doi.org/10.1016/j.ppnp.2015.01.002

[7] Sommerfeld, A. (1916) Zur quantentheorie der spektrallinien. Annalen der Physik, 356, 1-94. https://doi.org/10.1002/andp.19163561702

[8] Pohl, R., Gilman, R., Miller, G.A. and Pachucki, K. (2013) Muonic Hydrogen and the Proton Radius Puzzle. Annual Review of Nuclear and Particle Science, 63, 175-204. https://doi.org/10.1146/annurev-nucl-102212-170627

[9] Pohl, R., Antognini, A., Nez, F., Amaro, F.D., Biraben, F., Cardoso, J.M.R., Covita, D.S., Dax, A., Dhawan, S., Fernandes, L.M.P., Giesen, A., Graf, T., Hänsch, T.W., Indelicato, P., Julien, L., Kao, C.-Y., Knowles, P., Le Bigot, E.-O., Liu, Y.-W., Lopes, J.A.M., Ludhova, L., Monteiro, C.M.B., Mulhauser, F., Nebel, T., Rabinowitz, P., dos Santos, J.M.F., Schaller, L.A., Schuhmann, K., Schwob, C., Taqqu, D., Veloso, J.F.C.A. and Kottmann, F. (2010) The Size of the Proton. Nature, 466, 213-216. https://doi.org/10.1038/nature09250

[10] Antognini, A., Nez, F., Schuhmann, K., Amaro, F.D., Biraben, F., Cardoso, J.M.R., Covita, D.S., Dax, A., Dhawan, S., Diepold, M., Fernandes, L.M.P., Giesen, A., Gouvea, A.L., Graf, T., Hänsch, T.W., Indelicato, P., Julien, L., Kao, C.-Y., Knowles, P., Kottmann, F., Le Bigot, E.-O., Liu, Y.-W., Lopes, J.A.M., Ludhova, L., Monteiro, C.M.B., Mulhauser, F., Nebel, T., Rabinowitz, P., dos Santos, J.M.F., Schaller, L.A., Schwob, C., Taqqu, D., Veloso, J.F.C.A., Vogelsang, J. and Pohl, R. (2013) Proton Structure from the Measurement of $2 \mathrm{~s}-2 \mathrm{p}$ Transition Frequencies of Muonic Hydrogen. Science, 339, 417-420.

[11] Beyer, A., Maisenbacher, L., Matveev, A., Pohl, R., Khabarova, K., Grinin, A., Lamour, T., Yost, D.C., Hänsch, T.W., Kolachevsky, N. and Udem, T. (2017) The Rydberg Constant and Proton Size from Atomic Hydrogen. Science, 358, 79-85. https://doi.org/10.1126/science.aah6677

[12] Sick, I. (2003) On the Rms-Radius of the Proton. Physics Letters B, 576, 62-67. https://doi.org/10.1016/j.physletb.2003.09.092

[13] Rosenfelder, R. (2000) Coulomb Corrections to Elastic Electron Proton Scattering and the Proton Charge Radius. Physics Letters B, 479, 381-386. https://doi.org/10.1016/S0370-2693(00)00316-6

[14] Arrington, J. and Sick, I. (2015) Evaluation of the Proton Charge Radius from Electron-Proton Scattering. Journal of Physical and Chemical Reference Data, 44, Article ID: 031204. https://doi.org/10.1063/1.4921430

[15] Miller, G.A. (2019) Defining the Proton Radius: A Unified Treatment. Physical Review $C$, 99, Article ID: 035202. https://doi.org/10.1103/PhysRevC.99.035202

[16] Mohr, P.J., Newell, D.B. and Taylor, B.N. (2016) CODATA Recommended Values of the Fundamental Physical Constants: 2014. Journal of Physical and Chemical Reference Data, 45, Article ID: 043102. https://doi.org/10.1063/1.4954402

[17] Alexandrou, C., Constantinou, M., Hadjiyiannakou, K., Jansen, K., Kallidonis, C., 
Koutsou, G., Vaquero Avilés-Casco, A. and Wiese, C. (2017) Nucleon Spin and Momentum Decomposition Using Lattice QCD Simulations. Physical Review Letters, 119, Article ID: 142002. https://doi.org/10.1103/PhysRevLett.119.142002

[18] Li, Q., Xue, C., Liu, J.-P., Wu, J.-F., Yang, S.-Q., Shao, C.-G., Quan, L.-D., Tan, W.-H., Tu, L.-C., Liu, Q., Xu, H., Liu, L.-X., Wang, Q.-L., Hu, Z.-K., Zhou, Z.-B., Luo, P.-S., Wu, S.-C., Milyukov, V. and Luo, J. (2018) Measurements of the Gravitational Constant Using Two Independent Methods. Nature, 560, 582-588.

https://doi.org/10.1038/s41586-018-0431-5

[19] Luther, G.G. and Towler, W.R. (1982) Redetermination of the Newtonian Gravitational Constant G. Physical Review Letters, 48, 121-123. https://doi.org/10.1103/PhysRevLett.48.121

[20] Karagioz, V.P. and Izmailov, O.V. (1996) Measurement of the Gravitational Constant with a Torsion Balance. Measurement Techniques, 39, 979-987. https://doi.org/10.1007/BF02377461

[21] Gundlach, J.H. and Merkowitz, S.M. (2000) Measurement of Newton's Constant Using a Torsion Balance with Angular Acceleration Feedback. Physical Review Letters, 85, 2869-2872. https://doi.org/10.1103/PhysRevLett.85.2869

[22] Bagley, C.H. and Luther, G.G. (1997) Preliminary Results of a Determination of the Newtonian Constant of Gravitation: A Test of the Kuroda Hypothesis. Physical Review Letters, 78, 3047-3050. https://doi.org/10.1103/PhysRevLett.78.3047

[23] http://elpub.bib.uni-wuppertal.de/servlets/DerivateServlet/Derivate-429/d080201.pd $\underline{\mathrm{f}}$

[24] Armstrong, T.R. and Fitzgerald, M.P. (2003) New Measurements of G Using the Measurement Standards Laboratory Torsion Balance. Physical Review Letters, 91, Article ID: 201101. https://doi.org/10.1103/PhysRevLett.91.201101

[25] Luo, J., Hu, Z.-K., Fu, X.-H., Fan, S.-H. and Tang, M.-X. (1998) Determination of the Newtonian Gravitational Constant G with a Nonlinear-Fitting Method. Physical Review D, 59, Article ID: 042001. https://doi.org/10.1103/PhysRevD.59.042001

[26] Schlamminger, St., Holzschuh, E., Kündig, W., Nolting, F., Pixley, R.E., Schurr, J. and Straumann, U. (2006) Measurement of Newton's Gravitational Constant. Physical Review D, 74, Article ID: 082001. https://doi.org/10.1103/PhysRevD.74.082001

[27] Luo, J., Liu, Q., Tu, L.-C., Shao, C.-G., Liu, L.-X., Yang, S.-Q., Li, Q. and Zhang, Y.-T. (2009) Determination of the Newtonian Gravitational Constant G with Time-of-Swing Method. Physical Review Letters, 102, Article ID: 240801.

[28] Parks, H.V. and Faller, J.E. (2010) Simple Pendulum Determination of the Gravitational Constant. Physical Review Letters, 105, Article ID: 110801. https://doi.org/10.1103/PhysRevLett.105.110801

[29] Quinn, T., Parks, H., Speake, C. and Davis, R. (2013) Improved Determination of G Using Two Methods. Physical Review Letters, 111, Article ID: 101102. https://doi.org/10.1103/PhysRevLett.111.101102

[30] Rosi, G., Sorrentino, F., Cacciapuoti, L., Prevedelli, M. and Tino, G.M. (2014) Precision Measurement of the Newtonian Gravitational Constant Using Cold Atoms. Nature, 510, 518. https://doi.org/10.1038/nature13433

[31] Berg, E., Cross, W., Newman, R. and Bantel, M. (2014) A Measurement of G with a Cryogenic Torsion Pendulum. Philosophical Transactions of the Royal Society A, 372.

[32] Goedel, K. (2006) La prova matematica della esistenza di Dio. Bollati Boringhieri, Torino. 
[33] Wittgenstein, L. (2009) Tractatus logico-philosophicus e Quaderni 1914-1916. Piccola Biblioteca Einaudi Ns.

[34] Nico, J.S. (2009) Neutron Beta Decay. Journal of Physics G: Nuclear and Particle Physics, 36, Article ID: 104001. https://doi.org/10.1088/0954-3899/36/10/104001

[35] https://www.aps.org/policy/reports/multidivisional/neutrino/upload/main.pdf

[36] https://www.bipm.org/en/CGPM/db/26/1 